\title{
ALGEBRAS GENERATED BY A SUBNORMAL OPERATOR BY
}

\author{
ROBERT F. OLIN AND JAMES E. THOMSON ${ }^{1}$
}

\begin{abstract}
We use the notion of generalized Toeplitz operators to obtain some basic results concerning the $C^{*}$-algebra generated by a subnormal operator. We apply these results to problems concerning the intersection of $C^{*}(S)$ with rationally closed algebras generated by $S$. In particular, we prove that $C^{*}(S) \cap \mho(S)=\{f(S)$ : $\left.f \in R\left(\sigma_{\mathscr{W}(S)}(S)\right)\right\}$. The spectral inclusion property for generalized Toeplitz operators with symbols in $P^{\infty}(\mu)+C(\sigma(N))$ is also considered.
\end{abstract}

Throughout this paper $S$ will be a subnormal operator on a separable Hilbert space $\mathcal{H}$ with minimal normal extension $N$ on $\mathscr{K}$. The scalar spectral measure for $N$ will be denoted by $\mu$ and $P$ denotes the projection of $\mathcal{K}$ onto $\mathcal{H}$. For $f$ in $L^{\infty}(\mu)$ let $T_{f}$ denote the operator on $\mathcal{H}$ defined by

$$
T_{f} x=P f(N) x
$$

for each $x$ in $\mathcal{H}$. For obvious reasons $T_{f}$ is called a generalized Toeplitz operator. As in the classical case, where $S$ is a unilateral shift and $N$ is the bilateral shift, we shall be concerned with various problems that arise from the correspondence $f \in \Re \rightarrow T_{f}$ as $\mathscr{B}$ varies over different subalgebras of $L^{\infty}(\mu)$. (Consult [10] for an excellent account of the classical case.) In particular, our first algebra will be the continuous functions on the spectrum of $N$. Using this algebra, we will obtain some basic information concerning the $C^{*}$-algebra generated by a subnormal operator. We will apply these structure theorems to some problems concerning the intersection of this $C^{*}$-algebra with other algebras generated by $S$. Before we proceed, we need to introduce some notation.

If $\mathscr{B}$ is a Banach algebra with identity and $b \in \mathscr{B}$, then $\sigma_{\mathscr{B}}(b)$ denotes the spectrum of $b$. If $\mathscr{K}$ is a separable Hilbert space and $\mathscr{B}=\mathscr{B}(\mathcal{K})$, the algebra of bounded operators on $\mathscr{K}$, then $\sigma(T)$ will denote the spectrum of $T \in \mathscr{B}(\mathcal{K})$. The compact operators on $\mathscr{K}$ are denoted by $\mathcal{C}(\mathcal{K})$ and $\sigma_{e}(T)$ denotes the essential spectrum of $T$, i.e., the spectrum of $T$ in the Calkin algebra $\mathscr{B}(\mathcal{K}) / \mathcal{C}(\mathcal{K})$. The norm of $T$ in the Calkin algebra will be denoted by $\|T\|_{e}$. If $\Re$ is also a $C^{*}$-algebra, then $C^{*}(b)$ designates the $C^{*}$-algebra generated by $b$ and the identity. If $K$ is a compact subset of the complex plane, $\mathrm{C}$, then $C(K)$ represents the complex-valued continuous functions on $K$.

Received by the editors August 5, 1980 and, in revised form, March 13, 1981.

AMS (MOS) subject classifications (1970). Primary 47B20; Secondary 46L99, 47C15, 47D25.

'This research was supported in part by the National Science Foundation under Grant No. MCS7700966. 
Returning now to the generalized Toeplitz operators, we take our first subalgebra $\mathscr{G}$ to be $C(\operatorname{spt} \mu)=C(\sigma(N))$, where spt $\mu$ denotes the support of $\mu$. Let $\chi(z)=z$ for $z \in \mathbf{C}$. Using the Gelfand theory, we have that $C^{*}(N)$ is isometrically *-isomorphic to $C(\sigma(N))$, denoted $C^{*}(N) \cong C(\sigma(N))$, under the map $\Gamma$ that sends $N$ to $\chi$. Furthermore, for all $x, y$ in $\mathcal{K}$ and for all nonnegative integers $p$ and $m$ we have

$$
\left\langle S^{* p} S^{m} x, y\right\rangle=\left\langle S^{m} x, S^{p} y\right\rangle=\left\langle N^{m} x, N^{p} y\right\rangle=\left\langle P N^{* p} N^{m} x, y\right\rangle .
$$

Thus, it follows that $T_{\bar{\chi}^{p}} T_{\chi^{m}}=T_{\bar{\chi}^{p} \chi^{m}}$. A simple application of the Stone-Weierstrass theorem now shows that

$$
\left\{T_{f}: f \in C(\sigma(N))\right\} \subseteq C^{*}(S) .
$$

Let $\theta$ denote the induced map from $C^{*}(N)$ into $C^{*}(S)$. (Specifically, $\theta(f(N))=T_{f}$ for all $f \in C(\sigma(N))$.)

Using the work of Bunce [7], we shall exhibit a diagram that represents the cornerstone for the rest of the work in this paper. The commutator ideal of $C^{*}(S)$, denoted $I$, is the closed ideal generated by all elements of the form $A B-B A$, where $A, B \in C^{*}(S)$. It follows from [7] that

$$
\frac{C^{*}(S)}{I} \cong C\left(\sigma_{a}(S)\right)
$$

under the Gelfand map. (It is easy to show that the ideal $I$ in Corollary 11, [7], is the commutator ideal. Since the quotient of $C^{*}(S)$ with $\cap\left\{\varphi^{-1}(0): \varphi\right.$ a character $\}$ is abelian, it follows from an argument similar to that in [14, pp. 210-211] that $I$ is contained in this character ideal, because this character ideal is closed. Conversely, if $T \in C^{*}(S)$ and $T \notin I$, then there exists a character $\psi$ on $C^{*}(S) / I$ such that $\psi(T+I) \neq 0$. Clearly then there exists a character $\varphi$ on $C^{*}(S)$ such that $\varphi(T) \neq 0$.) If $\pi$ denotes the quotient map from $C^{*}(S)$ to $C^{*}(S) / I$ then we have induced a map $\tau: C(\sigma(N)) \rightarrow C\left(\sigma_{a}(S)\right)$ that makes the following diagram commute.

$$
\begin{array}{ccc}
C^{*}(N) & \cong & C(\sigma(N)) \\
\theta \downarrow & -\tau \\
C^{*}(S) & \underset{\pi}{\rightarrow} & \frac{C^{*}(S)}{I} \cong C\left(\sigma_{a}(S)\right)
\end{array}
$$

A well-known fact about subnormal operators is that $\sigma_{a}(S) \subset \sigma(N)$. It follows easily then that $\tau\left(\bar{\chi}^{l} \chi^{p}\right)$ is the restriction of $\bar{\chi}^{l} \chi^{p}$ to $\sigma_{a}(S)$ for all nonnegative integers $l$ and $p$. Applications of the Stone-Weierstrass and Tietze extension theorems yield that $\tau$ is the restriction map from $C(\sigma(N))$ onto $C\left(\sigma_{a}(S)\right)$. Using the properties of the maps in the commutative diagram above, we have the following theorem. (If $f$ is a function on $K$ and $L \subset K$ then $f \mid L$ denotes the restriction of $f$ to $L$.)

THEOREM 1. With the notation as above, $C^{*}(S) / I$ and $C\left(\sigma_{a}(S)\right)$ are isometrically isomorphic $C^{*}$-algebras with an isomorphism that sends $T_{f}+I$ to $f \mid \sigma_{a}(S)$ for each $f \in C(\sigma(N))$. Furthermore $T_{f} \in I$ if and only if $f \mid \sigma_{a}(S)$ is zero.

Theorem 1 describes the $C^{*}$-algebra, $C^{*}(S)$, modulo the commutator ideal. The following proposition describes the commutator ideal. 
Proposition 1. Let $T \in C^{*}(S)$ and let I denote the commutator ideal of $C^{*}(S)$. The following statements are equivalent.

a. $T \in I$.

b. For every $\lambda \in \sigma_{a}(S)$ and for every sequence $\left\{x_{n}\right\}$ of unit vectors in $\mathcal{H}$ such that $\left\|(S-\lambda) x_{n}\right\| \rightarrow 0$, it follows that $T x_{n} \rightarrow 0$.

c. For every $\lambda \in \sigma_{a}(S)$ and for some sequence $\left\{x_{n}\right\}$ of unit vectors in $\mathcal{H}$ such that $\left\|(S-\lambda) x_{n}\right\| \rightarrow 0$, it follows that $T x_{n} \rightarrow 0$.

Proof. Let $\lambda \in \sigma_{a}(S)$ and let $\left\{x_{n}\right\}$ be a sequence of vectors in $\mathcal{H}$ with $\left\|x_{n}\right\|=1$ and $\left\|(S-\lambda) x_{n}\right\| \rightarrow 0$. An easy computation now shows that

$$
\left(p\left(S, S^{*}\right)-p(\lambda, \bar{\lambda})\right) x_{n} \rightarrow 0
$$

for any polynomial $p(w, \bar{w})$ in two noncommuting variables.

Suppose now that $\varphi$ is a character on $C^{*}(S)$ with $\varphi(S)=\lambda$. Then [7] implies $\lambda \in \sigma_{a}(S)$. Using (1), we then have

$$
(T-\varphi(T)) x_{n} \rightarrow 0
$$

by the continuity of $\varphi$. Hence if $T \in I$, then $\varphi(T)=0$ and we have the conclusion of statement b. Clearly b implies c. Furthermore, if statement $\mathrm{c}$ is valid, then (2) implies $\varphi(T)=0$ for all characters $\varphi$ on $C^{*}(S)$. Hence $T \in I$.

In general, the problem of calculating the approximate point spectrum of a subnormal operator is difficult. Consult [13] for some general results on this problem. In our applications of Theorem 1 we will need to know that "peak points" are always in the approximate point spectrum of $S$. This can be made precise in the following way.

Proposition 2. Let $S$ be a subnormal operator on $\mathcal{H}$ with minimal normal extension $N$ on $\mathcal{K}$. Suppose $\lambda \in \sigma(N)$ is such that there exists $f \in C(\sigma(N))$ with the following properties.

a. $f(\lambda)=1$ and $|f(w)|<1$ for $w \in \sigma(S) \backslash\{\lambda\}$,

b. $f(N) \mathcal{H} \subset \mathcal{H}$.

Then $\lambda \in \sigma_{a}(S)$.

Proof. Using the methods in the proof of Theorem 1 in [17], we can choose a separating vector for the von Neumann algebra generated by $N$ that belongs to $\mathcal{H}$. Hence we can assume, without loss of generality, that $N=N_{1} \oplus N_{2}$ on $\mathscr{K}=L^{2}(\mu) \oplus$ $\mathscr{K}_{2} ; N_{1}$ is multiplication by $\chi$ on $L^{2}(\mu)$, and that $\mathscr{H} \supset H^{2}(\mu)$, the closure of the polynomials in $L^{2}(\mu)$. Hence $f(N)$ is multiplication by $f$ on $L^{2}(\mu)$.

Let $\Delta(\lambda, r)$ denote the open disc centered at $\lambda$ of radius $r$. Raise $f$ to a high enough power, say $p$, so that the supremum norm of $f^{p}$ outside $\Delta(\lambda, r)$ is less than $\frac{1}{4}$. Let $g=2 f^{p}$ and let $\varepsilon>0$. Since $\lambda \in \sigma(N)$ and $\sigma(N)=\operatorname{spt} \mu$, there exists a positive integer $m$ such that the $L^{2}(\mu)$ norm of $g^{m} \chi_{\Delta(\lambda, r)}$, denoted $\left\|g^{m} \chi_{\Delta(\lambda, r)}\right\|_{2}$, is bigger than 1 and that the supremum norm of $g^{m}$ outside $\Delta(\lambda, r)$ is less than $\varepsilon$. (Note that $m$ and $p$ depend on $r$.) Let

$$
q=\frac{g^{m}}{\left\|g^{m}\right\|_{2}}
$$


Then $q \in \mathcal{H},\|q\|=1$, and

$$
\begin{aligned}
\|(S-\lambda) q\|^{2} & =\int_{\Delta(\lambda, r) \cap \sigma(N)}|(z-\lambda) q|^{2} d \mu+\int_{\sigma(N) \Delta(\lambda, r)}|(z-\lambda) q|^{2} d \mu \\
& \leqslant r^{2}+\varepsilon^{2}\left(4\|N\|^{2}\right)\|\mu\| .
\end{aligned}
$$

The proof is finished if we choose $r$ and $\varepsilon$ sufficiently small.

In a wide class of examples (for example, if we assume $S$ is $k$-multicyclic [4]), the ideal $I$ in Theorem 1 is precisely $\mathcal{C}(\mathcal{H C})$.

COROLlaRy 1. Let $S$ be an irreducible subnormal operator with $S^{*} S-S S^{*} \in \mathcal{C}(\mathcal{H})$. Then $I=\mathcal{C}(\mathcal{H})$ and $\sigma_{a}(S)=\sigma_{e}(S)$. For $f, g \in C(\sigma(N))$ we have

a. $T_{f}$ is compact if and only if $f \mid \sigma_{e}(S)=0$,

b. $\left\|T_{f}\right\|_{e}=\left\|f \mid \sigma_{e}(S)\right\|_{\infty}$, where $\left\|T_{f}\right\|_{e}$ denotes the norm of $\pi\left(T_{f}\right)$ in the Calkin algebra and $\left\|f \mid \sigma_{e}(S)\right\|_{\infty}$ denotes the supremum norm of $f$ on $\sigma_{e}(S)$,

c. $T_{f g}-T_{f} T_{g}$ is a compact operator,

d. $\sigma_{e}\left(T_{f}\right)=f\left(\sigma_{e}(S)\right)$.

Proof. Since $S$ is an irreducible operator and $C^{*}(S)$ contains a nonzero compact operator (namely $S^{*} S-S S^{*}$ ), then [1, Corollary 2, p. 18] implies $\mathrm{C}(\mathcal{H}) \subset C^{*}(S)$. Furthermore, $C^{*}(S) / \mathcal{C}(\mathcal{H})$ is abelian, which implies $\mathcal{C}(\mathcal{H}) \supset I$.

Now $I$ is a nonzero closed two-sided ideal in $\mathcal{C}(\mathcal{H})$, so [1, Corollary 1, p. 18] implies that $I=\mathcal{C}(\mathcal{H})$. Hence we also have $\sigma_{a}(S)=\sigma_{e}(S)$ by an application of the Gelfand theory and the proof of Theorem 1. Part a of the corollary also follows from Theorem 1 and the fact $I=\mathcal{C}(\mathcal{H})$.

Similarly, since $I=\mathcal{C}(\mathcal{H})$ and $\sigma_{a}(S)=\sigma_{e}(S)$, part b follows from Theorem 1 and the definition of the norm on $C\left(\sigma_{e}(S)\right)$.

Now $\pi\left(T_{f} T_{g}\right)=\pi\left(T_{f}\right) \pi\left(T_{g}\right)$. Since $\pi\left(T_{h}\right)=\tau(h)$ for all $h \in C(\sigma(N))$, we have

$$
\pi\left(T_{f g}-T_{f} T_{g}\right)=\tau(f g)-\tau(f) \tau(g)=0
$$

because $\tau$ is multiplicative. This establishes part $\mathrm{c}$ of the corollary.

By the isomorphism in Theorem 1, we have that

$$
\sigma_{C^{*}(S) / \mathbb{C}(\mathscr{H})}\left(T_{f}\right)=f\left(\sigma_{e}(S)\right) .
$$

Part $\mathrm{d}$ is established if one uses [10, Theorem 4.28].

REMARKS. In an earlier draft of this paper, the universal diagram used in Theorem 1 appeared only in the proof of Corollary 2. After receiving the preprint [2], we realized the general context of Theorem 1 and Corollary 1 .

In the context of [2] the subnormal operator $S$ is multiplication by $z$ on $L_{a}^{2}(G)$, the square integrable (with respect to planar measure) analytic functions on $G$. Here $G$ is a bounded open connected subset of $\mathbf{C}$. By the proof of Lemma 6 in [20], one has that the commutant of $S$ consists of those multiplication operators, $M_{\psi}$, where $\psi$ is a bounded analytic function on $G$. It then follows easily that $S$ is irreducible because $G$ is connected. Using [5, Theorem 5.1], we see that $S$ has a compact self-commutator and, hence, $S$ satisfies the hypothesis of Corollary 1 .

The authors of [2] also characterize $\sigma_{e}(S)$. Using the notation of [2], we let $\partial_{2-r} G$ denote those points in $\partial G$ which are removable with respect to $L_{a}^{2}(G)$. Axler, 
Conway and McDonald show that $\sigma_{e}(S)$ equals $\partial_{2-e}(S)$, the set $\partial G \backslash \partial_{2-r} G$. We digress and indicate another proof of this result.

The easy part of the proof is to show $\sigma_{e}(S) \subset \partial_{2-e} G$. The proof of this containment given in [2] also establishes that the index of $S-\lambda$ is -1 whenever $S-\lambda$ is Fredholm and $\lambda \in \bar{G}$.

Now let $\lambda \notin \sigma_{e}(S)$. We want to show $\lambda \notin \partial_{2-e}(S)$. Without loss of generality we may assume $\lambda \in \partial G$. Hence index $(S-\lambda)=-1$. Therefore there exists an open neighborhood $V_{0}$ of $\lambda$ such that index $(S-\tau)=-1$ for all $\tau \in V_{0}$ (consult [10, Theorem 5.36 and Lemma 5.34]). Using [22], we choose a neighborhood $V_{1} \subset V_{0}$ for $\lambda$ such that for each $w \in V_{1}$ there exists a $K_{w}$ in the kernel of $S^{*}-\bar{w}$ with the property that the map $w \rightarrow K_{w}$ is coanalytic. We claim that $\left\langle 1, K_{\lambda}\right\rangle \neq 0$. Once this is established then there exists a neighborhood $V_{2} \subset V_{1}$ of $\lambda$ in which we may define the coanalytic function $\varphi(w)$ on $V_{2}$ by $\varphi(w)\left\langle K_{w}, 1\right\rangle=1$. Let $\tilde{K}_{w}=\varphi(w) K_{w}$. Then the map $w \rightarrow \tilde{K}_{w}$ for $w \in V_{2}$ is coanalytic; hence, for any fixed $f$ in $L_{a}^{2}(G)$ the map $w \rightarrow\left\langle f, \tilde{K}_{w}\right\rangle$ is analytic on $V_{2}$. Furthermore, for all $w \in G \cap V_{2}$ one can verify that for each $f \in L_{a}^{2}(G)$ one has $\left\langle f, \tilde{K}_{w}\right\rangle=f(w)$. (Use a Taylor series expansion of $f$ about $w$ and remember that $\tilde{K}_{w} \perp \operatorname{range}(S-w)$ and that $\left\langle 1, \tilde{K}_{w}\right\rangle=1$.) Hence $\lambda \notin \partial_{2-e} G$.

We need to show $\left\langle 1, K_{\lambda}\right\rangle \neq 0$ to finish the argument. Suppose to the contrary that 1 belongs to the range of $S-\lambda$. It follows then that $(z-\lambda)^{-1} \in L_{a}^{2}(G)$. It follows easily then that for every $r>0$ there exists a compact set $B_{r} \subset \Delta(\lambda, r) \backslash G$ such that $m\left(B_{r}\right)>0$. Here $m$ denotes Lebesgue measure on C. Using [11, Chapter II, Theorems 1.9 and 11.6 and p. 29], we have that there exist a point $z_{r} \in B_{r}$ and a continuous function $f$ on the Riemann sphere that is analytic on $(C \cup\{\infty\}) \backslash B_{r}$, satisfying $f\left(z_{r}\right)=1$ and $|f(w)|<1$ for $w \neq z_{r}$. Using Proposition 2, we now have that $z_{r} \in \sigma_{a}(S)$. It follows then that $\lambda \in \sigma_{a}(S)$ because $\sigma_{a}(S)$ is a closed set. Hence, $\lambda \in \sigma_{e}(S)$ by [18, Proposition 2.15]. This contradicts our assumption that $\lambda \notin \sigma_{e}(S)$.

Recall that the Banach space of bounded operators on a Hilbert space $\mathscr{K}$, denoted $\mathscr{B}(\mathscr{K})$, is the dual space of the trace class operators (with the trace class norm). If $T \in \mathscr{B}(\mathcal{K})$ and $K$ is a compact set containing $\sigma(T)$, then $\mathscr{R}(T, K)$ denotes the weak-star closed algebra generated by $\{r(T): r$ a rational function with poles off $K\}$. In particular, if $K$ is the polynomially convex hull [11] of $\sigma(T)$, then $\Re(T, K)$ is the weak-star closed algebra generated by $T$ and the identity, denoted $\mathscr{Q}(T)$. When $S$ is a subnormal operator, then $\mathcal{Q}(S)=\mathcal{W}(S)$, where $\mathcal{W}(S)$ is the weakly closed algebra generated by $S[17]$.

Using the same methods as the proof of [9, Theorem 2.1], one can show for a subnormal operator $S$ that $R(S, K)=\left\{f(S): f \in R^{\infty}(\mu, K)\right\}$. Here $\mu$ is the scalar spectral measure for the minimal normal extension $N$ of $S$ and $R^{\infty}(\mu, K)$ denotes the weak-star closure in $L^{\infty}(\mu)$ of the rational functions with poles off $K$. (Since $K \supset \sigma(S) \supset \sigma(N)$, then $K \supset$ spt $\mu$. Hence these rational functions are bounded on spt $\mu$. Of course, the weak-star topology on $L^{\infty}(\mu)$ referred to here is that inherited as the dual of $L^{1}(\mu)$.) In the particular case where $K$ is the polynomially convex hull of $\sigma(S), R^{\infty}(\mu, K)$ is the weak-star closure of the polynomials, which we will denote by $P^{\infty}(\mu)$. 
In the next part of the paper we will establish the following theorem.

THEOREM 2. Let $S$ be a subnormal operator. Then

$$
C^{*}(S) \cap \Re(S, K)=\left\{f(S): f \in R^{\infty}(\mu, K) \cap C(\operatorname{spt} \mu)\right\} .
$$

When $S$ is a normal operator, then this theorem is obvious if one uses the Gelfand theory and the characterization of $\Re(S, K)$ given in the previous paragraph. The result is also suggested by the diagram used in the proof of Theorem 1. (The diagram is the starting point for the proof of Theorem 2.)

The proof of Theorem 2 will rely on the results about $R^{\infty}(\mu, K)$ presented in [8]. In particular we will need an intrinsic characterization of the function algebra $R^{\infty}(\mu, K) \cap C(\operatorname{spt} \mu)$. This characterization is easy to understand in the polynomial case.

We will discuss the implications of Theorem 2 when $K$ is chosen to be the polynomially convex hull of $\sigma(S)$. With this choice of $K$ we have $R(S, k)=$ थ $S(S)$ $=\left\{f(S): f \in P^{\infty}(\mu)\right\}$. Using the notation of [19], we have $P^{\infty}(\mu)=L^{\infty}(\mu-\tilde{\mu}) \oplus$ $H^{\infty}$ (int $\left.\tilde{K}, \tilde{\mu}\right)$. Let $F$ denote the compact set $\tilde{K} \cup \operatorname{spt}(\mu-\tilde{\mu})$. It follows easily by the results in $[17,19]$ that $F=\sigma_{\mathscr{\partial S}(S)}(S)$. We let $R(L)$ denote the algebra obtained by taking the uniform closure of rational functions with poles off the compact set $L$. Using Theorem 2, we then have the following.

COROllary 2. Let $S$ be a subnormal operator. Then

$$
C^{*}(S) \cap \text { बiा }(S)=\left\{f(S): f \in R\left(\sigma_{\sigma_{\mathfrak{W}(S)}}(S)\right)\right\} .
$$

Proof. All that needs to be verified is that $P^{\infty}(\mu) \cap C(\operatorname{spt} \mu)=R(F)$. This, however, follows easily from [19] or [8, Chapter XIII, Theorem 1 and Chapter X, Theorem 6].

We will now discuss some of the results in [8] concerning $R^{\infty}(\mu, K)$ that are pertinent to our work here. Let $K$ be a compact subset of the plane and $\mu$ a nonzero measure on $K$. The envelope of $\mu$ with respect to the algebra $R(K)$, denoted $E(\mu, K)$, is the set of points $x \in \mathbf{C}$ possessing a complex representing measure $\mu_{x}$ for the algebra $R(K)$, that is absolutely continuous with respect to $\mu$, and satisfies $\mu_{x}(\{x\})=0$. The set of bounded Borel functions on $\mathbf{C}$ with compact support and zero $m_{E(\mu, K)}$ almost everywhere is denoted $B(E(\mu, K)$ ). (The restriction of planar Lebesgue measure $m$ to $E(\mu, K)$ is denoted $m_{E(\mu, K)}$. We note that $E(\mu, K)$ is a countable union of compact sets; hence, it is a Borel set.)

The set of functions of the form

$$
g(z)=\int g^{*}(x) \frac{1}{x-z} d m(x)
$$

with $g^{*}$ in $B(E(\mu, K))$ is denoted $A(\mu, K)$. One knows that $A(\mu, K)$ is an algebra of continuous functions on $\mathbf{C}$. Each function in $A(\mu, K)$ is zero at infinity, analytic on the interior of $E(\mu, K)$, denoted int $E(\mu, K)$, and analytic in a neighborhood of infinity. 
The algebra associated to the measure $\mu$ and the compact set $K$, denoted $\bar{A}(\mu, K)$, is the closure of the algebra $A(\mu, K)$ with respect to uniform convergence on the compact set $E(\mu, K)^{-} \cup(\operatorname{spt} \mu)$. Here $L^{-}$denotes the closure of the set $L$. The important results that we will need are the following.

(3) $\sigma_{R^{\infty}(\mu, K)}(\chi)=E(\mu, K)^{-} \cup(\operatorname{spt} \mu)$.

(4) The maximal ideal space of $\vec{A}(\mu, K)$ is $E(\mu, K)^{-} \cup($ spt $\mu)$.

(5) The set of nonpeak points of $\bar{A}(\mu, K)$ is precisely the set $E(\mu, K)$.

(6) Every function $f$ in $R^{\infty}(\mu, K)$ is a pointwise limit, $\mu$ almost everywhere, of a sequence $\left\{f_{n}\right\}_{1}^{\infty}$ of functions belonging to $\bar{A}(\mu, K)$ and satisfying $\left\|f_{n}\right\|_{\text {spt } \mu} \leqslant\|f\|_{\mu}$. Here $\|g\|_{L}$ denotes the supremum norm of a function $g$ on a set $L$, and $\|f\|_{\mu}$ denotes the $L^{\infty}(\mu)$ norm of $f$.

(7) If $h$ is a continuous function on spt $\mu$, then

$$
\inf _{f \in R^{\infty}(\mu, K)}\|h-f\|_{\mu}=\inf _{f \in \bar{A}(\mu, K)}\|h-f\|_{\text {spt } \mu} .
$$

Hence, $R^{\infty}(\mu, K) \cap C($ spt $\mu)=\bar{A}(\mu, K)$.

All these results may be found in Chapters 10 and 11 in [8]. It probably should be mentioned that $R\left(E(\mu, K)^{-} \cup(\operatorname{spt} \mu)\right) \subseteq \bar{A}(\mu, K)$ and strict containment may occur. (The algebras are the same when $K$ is chosen to be the polynomially convex hull of $\operatorname{spt} \mu$.)

We will also need the following function theoretic fact about $R^{\infty}(\mu, K)$.

Proposition 3. Let $\mu$ be a measure and $K$ a compact subset of $\mathbf{C}$ containing spt $\mu$. Let $L$ be a compact subset of $E(\mu, K)$ and let $U=\left((\operatorname{spt} \mu) \cup E(\mu, K)^{-}\right) \backslash$ L. Let $\iota$ denote the restriction map from $R^{\infty}(\mu, K)$ to $R^{\infty}\left(\mu_{U}, K\right)$. Then $\iota$ is an isometric isomorphism that is also a weak-star homeomorphism.

The following concept will be useful to us in the rest of the paper. If $z_{0} \in \mathbf{C}$, let $\Delta\left(z_{0}, 1 / n\right)=\left\{z \in \mathbf{C}:\left|z-z_{0}\right|<1 / n\right\}$ for each positive integer $n$. If $\mu$ is a measure and $f$ is a bounded $\mu$-measurable function then the essential range of $f$ at $z_{0}$ is the set

$$
\bigcap_{n=1}^{\infty} \sigma_{L^{\infty}\left(\mu_{\Delta(z 0,1 / n)}\right)}(f) \text {. }
$$

Proof of Proposition 3. Using the terminology in [8], we may assume that the residual part of $\mu$ is zero. (Equivalently, in the language used in [9], we may assume that $R^{\infty}(\mu, K)$ has no $L^{\infty}$ summand.) We shall show first that $\iota$ is an isometry.

Let $r \in R^{\infty}(\mu, K)$ and choose points $\lambda_{n} \in E(\mu, K)$ such that $\left|r\left(\lambda_{n}\right)\right| \rightarrow\|r\|_{\mu}$. That this choice is possible follows from Theorem 2, Chapter IV and Theorem 2 , Chapter I in [8]. Fix $n$ and let $\nu_{n}$ be a representing measure for $\bar{A}(\mu, K)$ at $\lambda_{n}$ that is carried by the peak points [11, Chapter II, Theorem 11.6]. By Lemma 12, Chapter 11 in [8], the restriction map from $A^{\infty}\left(\mu+\nu_{n}, K\right)$ to $R^{\infty}(\mu)$ is an onto isometric isomorphism. Here $A^{\infty}\left(\mu+\nu_{n}, K\right)$ is the weak-star closure of $\bar{A}(\mu, K)$ in $L^{\infty}\left(\mu+\nu_{n}\right)$.

We claim that for some peak point $z_{n}$, the essential range of $r$ at $z_{n}$ with respect to $\nu_{n}$ (hence, with respect to $\mu+\nu_{n}$ ) contains a point $x_{n}$ with $\left|x_{n}\right| \geqslant\left|r\left(\lambda_{n}\right)\right|$. If not, then $|r(w)|<\left|r\left(\lambda_{n}\right)\right|$ for almost all $w$ (with respect to $\nu_{n}$ ). Hence $\left|r\left(\lambda_{n}\right)\right|=$ $\left|\int r d \nu_{n}\right|<\left|r\left(\lambda_{n}\right)\right|$, a contradiction. 

have

Let $f_{n} \in \bar{A}(\mu, K)$ be a peak function at $z_{n}$. Then for all positive integers $m$, we

$$
\left\|f_{n}^{m} r\right\|_{\mu+\nu_{n}} \geqslant\left|r\left(\lambda_{n}\right)\right| \text {. }
$$

Noting that $\left\|f_{n}^{m} r \chi_{L}\right\|_{\mu+\nu_{n}} \rightarrow 0$ as $m \rightarrow \infty$, we can easily verify that for sufficiently large $m$,

$$
\left\|f_{n}^{m} r\right\|_{\mu_{U}} \geqslant\left|r\left(\lambda_{n}\right)\right|
$$

because of the isometry from $A^{\infty}\left(\mu+\nu_{n}, K\right)$ onto $R^{\infty}(\mu, K)$. Hence,

$$
\|r\|_{\mu_{U}} \geqslant\left|r\left(\lambda_{n}\right)\right| \text {. }
$$

Since this is true for all $n$, we have shown that $\iota$ is an isometry.

Let $F$ be a closed subset of $R^{\infty}(\mu, K)$ and let $B_{1}$ and $B_{2}$ be closed balls of the same radius with centers at the origins of $R^{\infty}(\mu, K)$ and $R^{\infty}\left(\mu_{U}, K\right)$, respectively. Noting that $\iota$ is weak-star continuous, $B_{1}$ is weak-star compact, and $\iota(F) \cap B_{2}=$ $\iota\left(F \cap B_{1}\right)$, we see that $\iota(F)$ is weak-star closed by the Krein-Smulian theorem. This shows that $\iota$ has closed range and is hence onto; and it also shows that $\iota^{-1}$ is continuous.

We are now ready to prove Theorem 2 . Since $R^{\infty}(\mu, K) \cap C($ spt $\mu)=\bar{A}(\mu, K)$, it suffices to establish the following equivalent version of Theorem 2 .

THEOREM 2'. Let $S$ be a subnormal operator. Then

$$
C^{*}(S) \cap \mathscr{R}(S, K)=\{f(S): f \in \bar{A}(\mu, K)\} .
$$

Proof. Let $f \in \bar{A}(\mu, K)$. Then $f \in C(\sigma(N))$ and $f(S)=T_{f}$ (notation as in Theorem 1). Hence $f(S) \in C^{*}(S) \cap \mathscr{Q}(S, K)$.

Conversely, suppose $T \in C^{*}(S) \cap \operatorname{R}(S, K)$. Then by Theorem 1 there exist $g \in C(K)$ (take any continuous extension of $g$ restricted to $\left.\sigma_{a}(S)\right)$ and $h \in R^{\infty}(\mu, K)$ such that $T=T_{h}$ and $T_{g-h} \in I$. We shall show $h \in \bar{A}(\mu, K)$.

First we show that the essential range of $g-h$ is $\{0\}$ at each peak point for $\bar{A}(\mu, K)$. Let $z_{0}$ be a peak point with $f$ a peaking function. Suppose $\lambda$ is a nonzero number belonging to the essential range of $g-h$ at $z_{0}$. Then $\lambda$ belongs to the essential range of $f^{n}(g-h)$ at $z_{0}$ for every positive integer $n$. Since $g-h=$ $\left(g-g\left(z_{0}\right)\right)-\left(h-g\left(z_{0}\right)\right)$, we may assume $g\left(z_{0}\right)=0$.

Let $\varepsilon>0$. Then for sufficiently large $n$ we have

$$
\varepsilon>\left\|f^{n} g\right\|_{E(\mu, K)} \cup(\text { spt } \mu) \text {. }
$$

Hence $\varepsilon>\left\|T_{f^{n} g}\right\|$ for large $n$. Now, by assumption, for every $n,|\lambda| \leqslant\left\|f^{n} h\right\|_{\mu}$ so that

$$
|\lambda| \leqslant\left\|T_{f^{n} h}\right\| \text {. }
$$

The last inequality follows from the fact that $f^{n} h \in R^{\infty}(\mu, K)$ and [16, Theorem 2.3]. Consequently, for every $n$, there exists $\lambda_{n} \in \sigma_{a}\left(T_{f^{n} h}\right)$ with $\left|\lambda_{n}\right| \geqslant|\lambda|$. Choose a sequence of unit vectors $\left\{x_{n}\right\}$ in $\mathcal{H}$ such that

$$
\left\|\left(T_{f^{n h}}-\lambda_{n}\right) x_{n}\right\|<\frac{1}{n} \text {. }
$$


Then

$$
\left\|T_{(h-g) f^{n}} x_{n}\right\| \geqslant\left\|T_{h f^{n}} x_{n}\right\|-\left\|T_{g f^{n}} x_{n}\right\| \geqslant|\lambda|-\frac{1}{n}-\varepsilon .
$$

Now define a sequence $\left\{y_{n}\right\}$ of unit vectors in $\mathcal{H}$ by setting

$$
y_{n}=\frac{f^{n}(S) x_{n}}{\left\|f^{n}(S) x_{n}\right\|} .
$$

Since $\left\|f^{n}(S) x_{n}\right\| \leqslant 1$ (recall $\left.\|f\| \leqslant 1\right)$ we have

$$
\left\|T_{h-g} y_{n}\right\| \geqslant|\lambda|-\frac{1}{n}-\varepsilon \text {. }
$$

We will now show that $\left\|\left(S-z_{0}\right) y_{n}\right\| \rightarrow 0$. Since $z_{0} \in \sigma_{a}(S)$ (this follows by Proposition 2), we will then have $\left\|T_{h-g} y_{n}\right\| \rightarrow 0$ because of Proposition 1. The last inequality will then imply $\lambda=0$, which contradicts our assumption.

Let $E$ denote the spectral measure for $N$. Then for any positive number $r$

$$
\begin{aligned}
\left\|\left(S-z_{0}\right) y_{n}\right\|^{2}= & \left\|\left(N-z_{0}\right) y_{n}\right\|^{2}=\int_{\Delta\left(z_{0}, r\right) \cap \sigma(N)}\left|z-z_{0}\right|^{2} d\left\langle E(z) y_{n}, y_{n}\right\rangle \\
& +\int_{\sigma(N) \Delta\left(z_{0}, r\right)}\left|f^{n}(z)\right|^{2}\left|z-z_{0}\right|^{2}\left(\frac{1}{\left\|f^{n}(S) x_{n}\right\|^{2}}\right) d\left\langle E(z) x_{n}, x_{n}\right\rangle .
\end{aligned}
$$

Now by inequality (8) we have

$$
\left\|f^{n}(S) x_{n}\right\| \geqslant \frac{|\lambda|-1 / n-\varepsilon}{\left\|T_{h-g}\right\|},
$$

so we may assume that the sequence $\left\{\left\|f^{n}(S) x_{n}\right\|\right\}$ is bounded below. Now choose $r$ so small so that the first integral in the last equality is small. Then choose $n$ sufficiently large so that $\left\|f^{n}\right\|_{\sigma(N) \backslash \Delta\left(z_{0}, r\right)}$ is arbitrarily small (hence, the second integral can be made small for large $n$ ).

Let $\varepsilon>0$. Since the essential range of $(g-h)$ at every point $z \in((\operatorname{spt} \mu) \cup \overline{E(\mu, K)}) \backslash E(\mu, K)$ is zero, for every such point $z$ there exists a positive integer $N(z)$ such that essential range of $(g-h)$ on $\Delta(z, 1 / N(z))$ is contained in $\Delta(0, \varepsilon)$. Hence there exists an open set $U(\varepsilon)$ containing the peak points of $\bar{A}(\mu, K)$ such that $\|g-h\|_{\mu_{U}} \leqslant \varepsilon$. Now by Proposition 3 and the results listed from [8] earlier, we have

$$
\begin{aligned}
\varepsilon & >\|g-h\|_{\mu_{U}} \geqslant \inf _{f \in R^{\infty}\left(\mu_{U}, K\right)}\|g-h\|_{\mu_{U}} \\
& \left.=\inf _{f \in \bar{A}(\mu, K)}\|g-f\|_{\mathrm{spt} \mu_{U}} \quad \text { (Proposition 3 implies } \bar{A}\left(\mu_{U}, K\right)=\bar{A}(\mu, K)\right) \\
& \geqslant \inf _{f \in \bar{A}(\mu, K)}\|g-f\|_{F},
\end{aligned}
$$

where $F$ is the closure of the peak points for $\bar{A}(\mu, K)$. Therefore $g$ restricted to $F$ belongs to the uniformly closed algebra $\left\{\left.f\right|_{F}: f \in \bar{A}(\mu, K)\right\}$.

Now let $r \in \bar{A}(\mu, K)$ be chosen such that $r|F=g| F$. Let $\varepsilon>0$ and choose an open set $G \supset F$ such that $\|r-g\|_{G}<\varepsilon$. Choose an open set $U$ such that

$$
\|g-h\|_{\mu_{U}}<\varepsilon
$$


and such that $\operatorname{spt}\left(\mu_{U}\right) \subset G$. Then $\|r-h\|_{\mu_{l}} \leqslant 2 \varepsilon$. Hence $|r(\lambda)-h(\lambda)| \leqslant 2 \varepsilon$ for all $\lambda \in E\left(\mu_{U}, K\right)=E(\mu, K)$. Since $\varepsilon$ is arbitrary $h \in \bar{A}(\mu, K)$.

Let $S$ be a pure subnormal operator on $\mathscr{H}$ with minimal normal extension $N$ on $\mathscr{K}$. (An operator $T$ is pure if it has no nonzero reducing subspace on which it is normal.) In his thesis, G. Keough [15] has considered the problem of when $\sigma(f(N)) \subset \sigma\left(T_{f}\right)$, i.e., spectral inclusion, holds when $f$ varies over different subalgebras of $L^{\infty}$. We shall address ourselves to this problem when the symbols of the generalized Toeplitz operators belong to the algebra $P^{\infty}(\mu)+C(\sigma(N))$. We note that this space of functions is a norm closed subalgebra of $L^{\infty}(\mu)$. Consult [8, Chapter 12, Theorem 2] or $[12, \S 26$, Theorem 26.4 and the second example].

THEOREM 3. Let $S$ be a pure subnormal operator. If $\sigma_{a}(S)=\sigma(N)$ then

a. $\sigma\left(T_{h}\right) \supset \sigma(h(N))$ for all $h \in P^{\infty}(\mu)+C(\sigma(N))$;

b. $\left\|T_{h}\right\|_{c}=\|h\|_{\infty}(=\|h(N)\|)$ for all $h \in P^{\infty}(\mu)+C(\sigma(N))$.

Hence the map $h \rightarrow T_{h}$ is an isometry on $P^{\infty}(\mu)+C(\sigma(N))$ and $T_{h}$ is not compact unless $h=0$.

In [15] it is shown that $\sigma\left(T_{h}\right) \supset \sigma(h(N))$ for all $h \in C(\sigma(N))$ if and only if $\sigma_{a}(S)=\sigma(N)$. Combining this fact with Theorem 3, we have the proof of the following

COROLlary 3. Let $S$ be a pure subnormal operator. The following are equivalent.

a. $\sigma_{a}(S)=\sigma(N)$.

b. $\sigma\left(T_{h}\right) \supset \sigma(h(N))$ for all $h \in P^{\infty}(\mu)+C(\sigma(N))$.

c. $\sigma\left(T_{h}\right) \supset \sigma(h(N))$ for all $h \in C(\sigma(N))$.

Proof of Theorem 3. Since $S$ is pure, there is no $L^{\infty}$ summand to $P^{\infty}(\mu)$. If we establish the theorem for the antisymmetric case then routine arguments yield the general case. (Consult [9] for this nomenclature.) Thus we assume that $P^{\infty}(\mu)=$ $H^{\infty}(G), G$ a simply connected open subset of the plane (consult [19] for the properties of $G$ ).

Let $h \in P^{\infty}(\mu)+C(\sigma(N))$. We shall show that $\sigma_{e}\left(T_{h}\right) \supset \sigma(h(N))$ from which a and $\mathrm{b}$ follow immediately. Noting that $\sigma(h(N))$ equals the union of the essential ranges of $\left.h\right|_{G \cap \sigma(N)}$ and $\left.h\right|_{\partial G}$, we shall establish the inclusion above for each of these essential ranges. We first deal with $\left.h\right|_{\sigma(N) \cap G}$. Since $\sigma_{e}\left(T_{h}\right)$ is closed, it is sufficient to show that $h(z) \in \sigma_{e}\left(T_{h}\right)$ for each $z$ in $\sigma(N) \cap G$.

Fix a point $z_{0}$ in $\sigma(N) \cap G$. Since $z_{0} \in \sigma_{a}(S)$ and $S$ is pure, there exists an orthonormal sequence $\left\{x_{n}\right\}$ of vectors such that $\lim \left\|\left(S-z_{0}\right) x_{n}\right\|=0$. As in the proof of Theorem 2, one can show that

$$
\lim \left\|\left(h(N)-h\left(z_{0}\right)\right) x_{n}\right\|=0,
$$

because $h$ is continuous at $z_{0}$. Hence,

$$
\lim \left\|\left(T_{h}-h\left(z_{0}\right)\right) x_{n}\right\|=0 .
$$

Thus $h\left(z_{0}\right) \in \sigma_{e}\left(T_{h}\right)$.

We now deal with the essential range of $\left.h\right|_{\partial G}$. Let $\sigma$ denote harmonic measure for a fixed point in $G$. Since $P^{\infty}(\mu)=H^{\infty}(G)$, it follows that $\mu_{\partial G} \ll \sigma$. Let $\lambda$ belong to 
the essential range of $\left.h\right|_{\partial G}$. Then there exists a sequence $\left\{E_{n}\right\}$ of Borel subsets of $\partial G$ with $\mu\left(E_{n}\right)>0$ and $\lim \sigma\left(E_{n}\right)=0$ (hence, $\lim \mu\left(E_{n}\right)=0$ ) such that $|h-\lambda|<\frac{1}{n}$ on $E_{n}$. We shall construct a sequence of vectors $\left\{x_{n}\right\}$ in $\mathcal{H}$ such that $x_{n} \rightarrow 0$ weakly, $1 \leqslant\left\|x_{n}\right\| \leqslant 2$ and $\left\|\left(T_{h}-\lambda\right) x_{n}\right\| \rightarrow 0$. That implies that $\lambda \in \sigma_{e}\left(T_{h}\right)$ and thus will complete the proof of Theorem 3.

As in the beginning of the proof of Proposition 2, we can assume, without loss of generality, that $N=N_{1} \oplus N_{2}$ on $\mathscr{K}=L^{2}(\mu) \oplus \mathcal{K}_{2}$; the scalar spectral measure for $\mathrm{N}_{2}$ is absolutely continuous with respect to $\mu$; and that $\mathcal{H} \supset H^{2}(\mu)$, the closure of the polynomials in $L^{2}(\mu)$. We also assume that $\mu$ is a probability measure.

Let $\psi_{n} \in P^{\infty}(\sigma)$ satisfy

$$
\left|\psi_{n}(z)\right|= \begin{cases}\frac{1}{n}, & z \in \partial G \backslash E_{n}, \\ \frac{1}{\sqrt{\mu\left(E_{n}\right)}}, & z \in E_{n} .\end{cases}
$$

(This is an application of Szegö's theorem for $H^{\infty}(\sigma)$.) Then

$$
\int_{\partial G}\left|\psi_{n}\right|^{2} d \mu \leqslant \frac{1}{n^{2}} \int_{\partial G \backslash E_{n}} d \mu+\int_{\mu\left(E_{n}\right)} d \mu \leqslant \frac{1}{n^{2}}+1 .
$$

We also have

$$
\int_{\partial G}\left|\psi_{n}\right|^{2} d \mu \geqslant \int_{E_{n}}\left|\psi_{n}\right|^{2} d \mu=1 .
$$

Let $\varphi$ be the conformal map of $G$ onto $D$, the open unit disc, and extend $\varphi$ to $P^{\infty}(\sigma)$ via [19, Lemma 4.4]. Multiplying $\psi_{n}$ by an appropriate power of $\varphi$, we may (and do) assume that

$$
2 \geqslant \int_{\bar{G}}\left|\psi_{n}\right|^{2} d \mu=\left\|\psi_{n}\right\|^{2} \geqslant 1
$$

and

$$
\int_{G}\left|\psi_{n}\right|^{2} d \mu \leqslant \frac{1}{n} .
$$

It follows easily that $\psi_{n} \rightarrow 0$ weakly in $L^{2}(\mu)$; hence, they converge weakly to zero in $\mathcal{H}$. We also have

$$
\begin{aligned}
\left\|\left(T_{h}-\lambda\right) \psi_{n}\right\|^{2} & \leqslant\left\|(h(N)-\lambda) \psi_{n}\right\|^{2} \\
& \leqslant\|h-\lambda\|_{\infty} \int_{G}\left|\psi_{n}\right|^{2} d \mu+\frac{1}{n^{2}}\|h-\lambda\|_{\infty} \int_{\partial G \backslash E_{n}} d \mu+\frac{1}{n^{2}} \int_{E_{n}} \frac{1}{\mu\left(E_{n}\right)} d \mu \rightarrow 0
\end{aligned}
$$

as $n \rightarrow \infty$. This finishes the proof.

If $S$ is a pure subnormal operator with $\sigma_{e}(S)=\sigma(N)$ one might hope that more than Theorem 3 is true, one might want $\sigma(h(N)) \subset \sigma\left(T_{h}\right)$ for all $h \in L^{\infty}(\mu)$. However, this can be false as seen by Example 6.2, Chapter III, [15]. (The subnormal 
operator in this example does not have a compact self-commutator.) If spectral inclusion is valid, then for any compact operator $K \in \mathscr{G}(\mathcal{H})$

$$
\left\|T_{h}+K\right\| \geqslant\|h(N)\|=\left\|T_{h}\right\|
$$

for all $h \in L^{\infty}(\mu)$. (Consult [15, Chapter IV, or 2.4].) This leads us to the weaker question, if $S$ is a pure subnormal with $\sigma_{e}(S)=\sigma(N)$ and $S^{*} S-S S^{*} \in \mathcal{C}(\mathcal{H})$, can $T_{h} \in \mathcal{E}(\mathcal{H})$ for a nonzero $h \in L^{\infty}(\mu)$ ? The answer is yes.

EXAMPLE. Let $\bar{D}$ denote the closed unit disc and let $C$ be a nowhere dense compact subset of $D$ with positive area. We choose a sequence $\left\{D_{n}\right\}$ of disjoint open discs in $D \backslash C$ with each disc $D_{n}$ having radius $r_{n}$ and center $\lambda_{n}$ such that

$$
\operatorname{int}\left(D \backslash \cup D_{n}\right)=\varnothing
$$

and

$$
r_{n}<d_{n}^{2} 2^{-n},
$$

where $d_{n}=\operatorname{dist}\left\{\lambda_{n}, C\right\}$. Let $K$ be the Swiss cheese $\bar{D} \backslash \cup D_{n}$. Note int $K=\varnothing$. Put $\Gamma=\partial D \cup\left(\cup \partial D_{n}\right)$ and let $d s$ denote arc length on $\Gamma$. Since $\Sigma r_{n}<\infty$, we have $\int_{\Gamma} d s<\infty$. Let $d \mu$ be the sum of area measure restricted to $C$ and $d s$. Let $R^{2}(d s)$ $\left(R^{2}(d \mu)\right)$ be the $L^{2}$ closure of $R(K)$ with respect to $d s(d \mu)$. Let $S_{1}\left(S_{2}\right)$ be multiplication by $z$ on $R^{2}(d s)\left(R^{2}(d \mu)\right)$. Hence $S_{2}^{*} S_{2}-S_{2} S_{2}^{*}$ is compact [4].

By Cauchy's theorem we have

$$
\int_{\cup \partial D_{n}} \frac{d z}{d s} \cdot f d s-\int_{\partial D} \frac{d z}{d s} \cdot f d s=0
$$

for all rational functions $f$ with poles off $K$; hence, $S_{1}$ is pure because $|d z / d s|>0$ almost everywhere [3, Proposition 3.10]. Using Cauchy's theorem again, we have for every rational function $f$ with poles off $K$ and for every $z_{0} \in C$,

$$
\left|f\left(z_{0}\right)\right| \leqslant \frac{1}{2 \pi} \int_{\Gamma} \frac{|f(z)||d z|}{\left|z-z_{0}\right|} \leqslant \frac{1}{2 \pi}\|f\|_{2}\left(\sum_{n} \frac{2 \pi r_{n}}{d_{n}^{2}}\right)^{1 / 2} \leqslant M_{1}\|f\|_{2},
$$

where $M_{1}$ is a positive constant (independent of $z_{0} \in C$ ). Hence if $f$ is a rational function with poles off $K$ there exist positive constants $M_{2}$ and $M_{3}$ such that

$$
\|f\|_{d s} \leqslant M_{2}\|f\|_{d \mu} \leqslant M_{3}\|f\|_{d s}
$$

(where all norms are taken in the indicated $L^{2}$ space). Hence $S_{1}$ and $S_{2}$ are similar; thus, $S_{2}$ is pure [21]. It follows that $\sigma\left(S_{2}\right)=\sigma_{a}\left(S_{2}\right)=\sigma_{e}\left(S_{2}\right)=\sigma(N)$.

Using (11) and (12), we see there exists a positive constant $M_{4}$ such that for each $z_{0} \in C$, there exists $K_{z_{0}} \in R^{2}(d \mu)$ with $\left\|K_{z_{0}}\right\| \leqslant M_{4}$ so that

$$
\left\langle f, K_{z_{0}}\right\rangle=f\left(z_{0}\right)
$$

for each $f \in R(K)$. Let $h$ be the characteristic function of $C$. Let $g_{n} \rightarrow 0$ weakly in $R^{2}(d \mu)$. Then clearly $\left(h g_{n}\right)(z)=0$ for all $z \notin C$ and by (13), we also have $\left(h g_{n}\right)(z)$ $\rightarrow 0$ for all $z \in C$. Since

$$
\left|\left(h g_{n}\right)(z)\right| \leqslant M_{4} \sup \left\|g_{n}\right\|_{2},
$$

it follows by the Lebesgue dominated convergence theorem that $T_{h}$ is compact. 


\section{BIBLIOGRAPHY}

I. W. Arveson, An invitation to $C^{*}$-algebra, Graduate Texts in Math., Springer-Verlag, New York, 1976.

2. S. Axler, J. B. Conway and G. McDonald, Toeplitz operators on Bergman spaces, preprint.

3. J. Ball, R. Olin and J. Thomson, Weakly closed algebras of subnormal operators, Illinois J. Math. 22 (1978), 315-326.

4. C. A. Berger and B. I. Shaw, Selfcommutators of multicyclic hyponormal operators are trace class, Bull. Amer. Math. Soc. 79 (1973), 1193-1199.

5. , Hyponormality: its analytic consequences, Amer. J. Math. (to appear).

6. J. Bram, Subnormal operators, Duke Math. J. 22 (1955), 75-94.

7. J. Bunce, Characters on singly generated $C^{*}$-algebras, Proc. Amer. Math. Soc. 25 (1970), 297-303.

8. J. Chaumat, Adhérence faible étoile d'algèbres de fractions rationnelles, Publ. Math. d'Orsay No. 147, U.E.R. Math., Univ. de Paris XI, Orsay, 1975.

9. J. B. Conway and R. Olin, A functional calculus for subnormal operators. II, Mem. Amer. Math. Soc. No. 184 (1977).

10. R. G. Douglas, Banach algebra techniques in operator theory, Academic Press, New York, 1972.

11. T. Gamelin, Uniform algebras, Prentice-Hall, Englewood Cliffs, N. J., 1969.

12. , Rational approximation theory, Course Notes, 1975.

13. W. Hastings, The approximation point spectrum of a subnormal operator, preprint.

14. I. N. Herstein, Topics in algebra, Blaisdell, Waltham, Mass., 1964.

15. G. Keough, Subnormal operators, Toeplitz operators, and the spectral inclusion theorem, Indiana Univ. Ph. D. Thesis, 1979.

16. R. Olin, Functional relationships between a subnormal operator and its minimal normal extension, Pacific J. Math. 63 (1976), 221-229.

17. R. Olin and J. Thomson, Algebras of subnormal operators, J. Funct. Anal. 37 (1980), 271-301.

18. C. Pearcy, Some recent developments in operator theory, CBMS Regional Conf. Ser. in Math., no. 36, Amer. Math. Soc., Providence, R. I., 1978.

19. D. Sarason, Weak-star density of the polynomials, J. Reine Angew. Math. 252 (1972), 1-15.

20. A. L. Shields and L. J. Wallen, The commutants of certain Hilhert space operators, Indiana Univ. Math. J. 20 (1971), 777-788.

21. J. G. Stampfli, Analytic extensions and spectral localization, J. Math. Mech. 16 (1966), 287-296.

22. M. A. Subin, Factorization of parameter-dependent matrix functions in normal rings and certain related questions in the theory of Noetherian operators, Mat. Sb. 73 (1967), 610-629; English transl. in Math. USSR-Sb. 2 (1967), 543-560.

Department of Mathematics, Virginia Polytechnic Institute and State University, BlacksBURG, VIRGINIA 24061 